\title{
Junge Forscher überzeugen mit innovativen Lösungsansätzen
}

\section{Die 14. VOCO Dental Challenge in Cuxhaven wurde ihrem Namen erneut mehr als gerecht: 9 junge Wissenschaftler traten bei dem renommierten Forschungswettbewerb in einen anspruchsvollen Wettstreit auf höchstem Niveau.}

Es wurde eine beeindruckende Themenvielfalt aufgeboten, die sowohl Antworten auf komplexe Fragestellungen lieferte als auch neue Fragen aufwarf, die eine nähere Betrachtung verlangen, und dann der un-

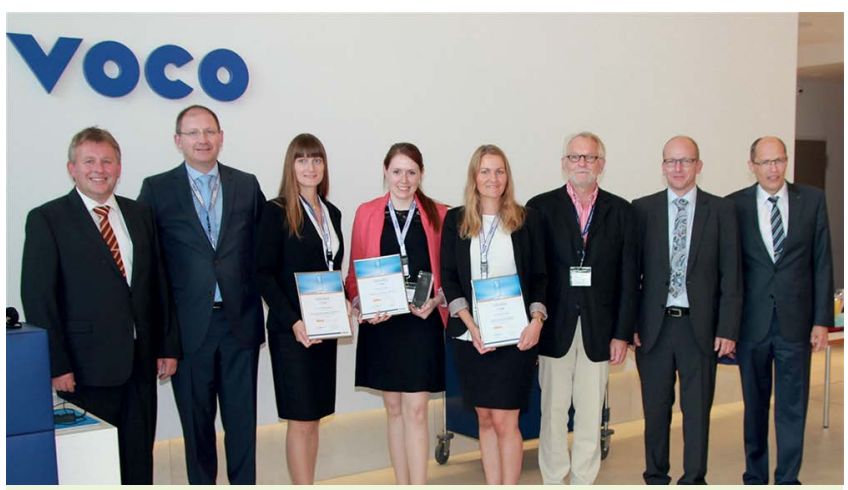

Die Preisträgerinnen der diesjährigen VOCO Dental Challenge zusammen mit M. Plaumann (rechts), Dr. M. Danebrock (Wissenschaftlicher Service, links) und der Jury. abhängigen und hochkarätig besetzten Jury gegenüber beantwortet werden mussten. Dieser gehörten in diesem Jahr Prof. Dr. Christian Apel (Universität Aachen), Prof Dr.-Ing. Dr. Martin Rosentritt (Universität Regensburg) sowie Prof. (UH) Dr. Wolf-Dieter Müller (Charité Berlin) an. VOCO Geschäftsführer Manfred Thomas Plaumann formulierte die Idee hinter dem Forschungswettbewerb so: „Die Forschungslandschaft braucht die Impulse und bisweilen auch das Querdenken ihrer jungen Wissen- schaftler. Die VOCO Dental Challenge lenkt den Blick auf den Nachwuchs der Branche." Für VOCO als Partner der Hochschulen sei es wichtig, ,den Nachwuchs zu ermutigen, sein großes Potenzial auszuschöpfen“. Plaumann freute sich besonders über die hohen Teilnehmerzahlen und das herausragende Engagement der Bewerber, die es in die Finalrunde geschafft haben - spricht dies doch für das Renommee der Dental Challenge.Den 1. Platz belegte in diesem Jahr Dr. Eva Maier (Universität Erlangen-Nürnberg), die mit ihrem Vortrag zum Thema „All-in-one“Silanisierung - geht das?“ die Jury und das Auditorium beeindruckte. Platz 2 ging an Dr. Marta Lungova (Universität Göttingen). Sie überzeugte mit ihrem Beitrag zum Thema „Functionalized hybrid silica nanoparticles in dental adhesive: bioactivity and physiochemical properties“. Den 3. Platz sicherte sich Bettina Hanßen(Universität Leipzig). Sie hatte sich erfolgreich der „Bewertung von Klasse-V-Kompositfüllungen - klinisch und mit quantitativer Randanalyse“ gewidmet.

Die Preisträgerinnen und ihr unterstützendes Team erhalten Preisgelder in Höhe von 6000, 4000 bzw. $2000 €$. Zudem gibt es Publikationszuschüsse von jeweils $2000 €$ zur Unterstützung ihrer weiteren Arbeit.

Aus einer Pressemitteilung der vOCO $\mathrm{GmbH}$, Cuxhaven www.voco.de 